Теорія Ймовір. та Матем. Статист. Вип. 82, 2010
Theor. Probability and Math. Statist.

No. 82, 2011, Pages 139-148

S 0094-9000(2011)00833-2

Article electronically published on August 5, 2011

\title{
INTERPOLATION OF A HOMOGENEOUS, SPACE-ISOTROPIC, AND TIME-STATIONARY RANDOM FIELD FROM OBSERVATIONS ON AN INFINITE CYLINDRICAL SURFACE. I
}

UDC 519.21

\author{
N. SEMENOVS'KA
}

\begin{abstract}
We solve the problem of interpolation of a homogeneous, space-isotropic, and time-stationary random field in the case of a finite sample observed on an infinite cylindrical surface. An explicit formula for the corresponding mean square error of interpolation is obtained. The asymptotic behavior of the error is studied as the number of observations is increasing. Conditions for the error-free approximation are given. For the problem of the error-free approximation, we find an optimal distribution of the weight coefficients in the interpolation formula.
\end{abstract}

\section{INTRODUCTION}

Let $\xi(x, t), x=\left(r, \varphi_{1}, \ldots, \varphi_{n-1}\right)$, be a homogeneous random field in the $(n+1)$ dimensional Euclidean space $\mathbb{R}^{n+1}$. We assume that $\xi$ is isotropic with respect to the spatial coordinates $r, \varphi_{1}, \ldots, \varphi_{n-1}$ and that it is stationary with respect to time $t$. This means that $\mathrm{E} \xi(x, t)=$ const (in what follows we assume that $\mathrm{E} \xi(x, t)=0$ ) and the covariance function

$$
\varphi(r, \tau)=\mathrm{E} \xi(x, t) \overline{\xi(y, s)}
$$

depends on $x$ and $y$ via the distance $r=|x-y|$ as well as depending on $s$ and $t$ via the distance $\tau=|t-s|$.

The following spectral decompositions are proved in [1] for the covariance function if the field is homogeneous, space-isotropic, and time-stationary:

$$
\varphi(r, \tau)=2^{(n-2) / 2} \Gamma\left(\frac{n}{2}\right) \int_{0}^{\infty} \int_{-\infty}^{\infty} \frac{J_{\frac{n-2}{2}}(\lambda r)}{(\lambda r)^{(n-2) / 2}} e^{i \tau \mu} d \Phi(\lambda, \mu),
$$

where $J_{\nu}(z)$ is the Bessel function of the first kind and of order $\nu$ and where $\Phi(\lambda, \mu)$ is a bounded and nondecreasing function with respect to every argument. A similar decomposition holds for the field itself:

$$
\xi(x, t)=c_{n} \sum_{m \geq 0} \sum_{l=1}^{h(m, n)} S_{m}^{l}(\bar{\varphi}) \int_{-\infty}^{\infty} e^{i t \mu} \zeta_{m}^{l}(r, \mu) d \mu
$$

(see [1), where $c_{n}^{2}=2^{n-1} \Gamma(n / 2) \pi^{n / 2}, S_{m}^{l}(\bar{\varphi})$ are the so-called orthonormal spherical harmonics of order $m$,

$$
h(m, n)=(2 m+n-2) \frac{(m+n-3) !}{(n-2) ! m !}
$$

2010 Mathematics Subject Classification. Primary 60G60.

Key words and phrases. Isotropic random field, interpolation, optimal estimates. 
is the total number of spherical harmonics of order $m, \zeta_{m}^{l}(r, \mu)$ are defined by

$$
\zeta_{m}^{l}(r, \mu)=\int_{0}^{\infty} \frac{J_{m+\frac{n-2}{2}}(\lambda r)}{(\lambda r)^{(n-2) / 2}} Z_{m}^{l}(d \lambda, d \mu)
$$

and where $Z_{m}^{l}(\cdot)$ is a sequence of noncorrelated random measures defined on Borel sets of $(0,+\infty) \times(-\infty,+\infty)$. The random measures $Z_{m}^{l}(\cdot)$ correspond to the spectral measure $\Phi$ and are such that

$$
\begin{gathered}
\mathrm{E} Z_{m}^{l}(S)=0, \\
\mathrm{E} Z_{m}^{l}\left(S_{1}\right) \overline{Z_{p}^{q}\left(S_{2}\right)}=\delta_{m}^{p} \delta_{l}^{q} \Phi\left(S_{1} \cap S_{2}\right)
\end{gathered}
$$

for all Borel sets $S, S_{1}$, and $S_{2}$ belonging to $(0,+\infty) \times(-\infty,+\infty)$.

It follows from equalities (44) that $\zeta_{m}^{l}(r, \mu)$ are noncorrelated random variables for different $m$ and $l$, namely

$$
\begin{gathered}
\mathrm{E} \zeta_{m}^{l}(r, \mu) \overline{\zeta_{p}^{q}(r, \mu)}=\delta_{m}^{p} \delta_{l}^{q} b_{m}(r, \mu), \\
b_{m}(r, \mu)=\int_{0}^{\infty} \frac{J_{m+\frac{n-2}{2}}^{2}(\lambda r)}{(\lambda r)^{n-2}} d \Phi(\lambda, \mu) .
\end{gathered}
$$

The above fields are called space-isotropic and time-stationary. We study the problem of the best linear interpolation constructed from a finite set of observations after the field on an infinite cylindrical surface.

The extrapolation problem is studied in 2 for space-isotropic and time-stationary random fields in the case of observations on an infinite cylindrical surface. The interpolation problem is considered in [3] and [4] for a homogeneous and isotropic field in the case of observations on a sphere (the interpolation problem is considered in [3] and [4] for both cases of the center of the sphere and of an arbitrary point inside the sphere in the $n$-dimensional space).

In what follows we assume that the field possesses the spectral density $f(x, y)$, which means that

$$
\Phi(\lambda, \mu)=\int_{0}^{\lambda} \int_{-\infty}^{\mu} f(x, y) d x d y
$$

\section{EXTRAPOLATION OF A RANDOM FIELD FROM OBSERVATIONS ON AN INFINITE CYLINDRICAL SURFACE}

Denote by

$$
E=\left\{(x, t): r=\text { const, } 0 \leq \varphi_{1}<2 \pi, 0 \leq \varphi_{i}<\pi, i=2, \ldots, n-1,-\infty<t<\infty\right\}
$$

an infinite cylindrical surface and let $H_{\xi}(E)$ be a closed in the mean square sense linear manifold generated by $\xi(x, t),(x, t) \in E$.

Assume that the field $\xi(x, t)$ is observed at every point $(x, t) \in E$. The best linear extrapolation problem for $\xi(y, s), y=(\rho, \bar{\psi}), \bar{\psi}=\left(\psi_{1}, \psi_{2}, \ldots, \psi_{n-1}\right)$, reduces to finding the projection $\widehat{\xi(y, s)}$ of the element $\xi(y, s)$ to the subspace $H_{\xi}(E)$. This projection is unique and can be found from the following condition:

$$
\mathrm{E} \xi(y, s) \overline{\xi(x, t)}=\mathrm{E} \widehat{\xi(y, s)} \overline{\xi(x, t)} \quad \text { for all }(x, t) \in E .
$$

It is known from the paper [2] that the best linear extrapolation for a space-isotropic and time-stationary random field $\xi(y, s)$ constructed from observations on an infinite cylindrical surface is given by

$$
\widehat{\xi(y, s)}=c_{n} \sum_{m \geq 0} \sum_{l=1}^{h(m, n)} S_{m}^{l}(\bar{\psi}) \int_{-\infty}^{\infty} \frac{b_{m}(r, \rho, \mu)}{b_{m}(r, \mu)} e^{i s \mu} \zeta_{m}^{l}(r, \mu) d \mu,
$$


where

$$
\begin{gathered}
b_{m}(r, \rho, \mu)=\int_{0}^{\infty} \frac{J_{m+\frac{n-2}{2}}(\lambda r)}{(\lambda r)^{(n-2) / 2}} \cdot \frac{J_{m+\frac{n-2}{2}}(\lambda \rho)}{(\lambda \rho)^{(n-2) / 2}} f(\lambda, \mu) d \lambda, \\
b_{m}(r, \mu)=b_{m}(r, r, \mu) .
\end{gathered}
$$

Note that the right-hand side of interpolation formula (7) is expressed in terms of the sequence of random measures $Z_{m}^{l}$. From the point of view of applications, it is more useful to rewrite formula (7) in terms of values of the field $\xi(x, t),(x, t) \in E$, since this is what we observe. Such an interpolation formula is given by

$$
\widehat{\xi(y, s)}=\int_{S_{n}} \int_{-\infty}^{\infty} C(y, s, x, t) \xi(x, t) d \mu_{n} d t
$$

(see [2]), where

$$
C(y, s, x, t)=\frac{1}{2 \pi \omega_{n}} \sum_{m \geq 0} \frac{2 m+n-2}{n-2} C_{m}^{(n-2) / 2}(\cos \theta) \int_{-\infty}^{\infty} e^{i(s-t) \mu} \frac{b_{m}(r, \rho, \mu)}{b_{m}(r, \mu)} d \mu
$$

and where $C_{m}^{\nu}(z)$ are the Gegenbauer polynomials defined as the coefficients of the decomposition of the generating function

$$
\left(1-2 t z+t^{2}\right)^{-\nu}=\sum_{m=0}^{\infty} C_{m}^{\nu}(z) t^{m} .
$$

In equality (10), $\theta$ is the angle between the vectors $x$ and $y, \mu_{n}$ is the Lebesgue measure on a unit sphere of $\mathbb{R}^{n}$, and $\omega_{n}=2 \pi \frac{\pi^{(n-2) / 2}}{\Gamma(n / 2)}$ denotes the area of the surface of this sphere.

The corresponding error of interpolation $\sigma^{2}=\mathrm{E}(\xi(y, s)-\widehat{\xi(y, s)})^{2}$ is equal to

$$
\sigma^{2}=\varphi(0,0)-\frac{c_{n}^{2}}{\omega_{n}} \cdot \sum_{m \geq 0} h(m, n) \int_{-\infty}^{\infty} \frac{b_{m}^{2}(r, \rho, \mu)}{b_{m}(r, \mu)} d \mu
$$

\section{An Approximation of the InTEGRAL $\int_{S_{n}} \int_{-\infty}^{\infty} C(y, s, x, t) \xi(x, t) d \mu_{n} d t$ BY ITS INTEGRAL SUMS}

When applying formula (9) in practice, the integral

$$
\int_{S_{n}} \int_{-\infty}^{\infty} C(y, s, x, t) \xi(x, t) d \mu_{n} d t
$$

is approximated by the corresponding integral sum. This means that the interpolation in the space

$$
H_{\xi}(E)=C l\left\{\sum \alpha_{k} \xi\left(x_{k}, t_{k}\right),\left(x_{k}, t_{k}\right) \in E\right\}
$$

is reduced to the interpolation of the random variable $\xi(y, s)$ constructed from observations in the space

$$
H_{X}(E)=\left\{\sum \alpha_{k} \xi\left(x_{k}, t_{k}\right),\left(x_{k}, t_{k}\right) \in X\right\},
$$

where $X=\left\{\left(x_{1}, t_{1}\right), \ldots,\left(x_{N}, t_{N}\right)\right\}$ is a certain finite set of observations on an infinite cylindrical surface $E$.

According to this method, the integral (9) is replaced by a finite linear combination (13) of values $\xi\left(x_{k}, t_{k}\right)$ at points $\left(x_{k}, t_{k}\right)$ of a certain set $X$. Thus a problem arises on the accuracy of this replacement and, as a consequence, on the accuracy of the interpolation formula (9). 
Fix a set $X=\left\{\left(x_{1}, t_{1}\right), \ldots,\left(x_{N}, t_{N}\right)\right\}$. Since $H_{X} \subset H_{\xi}$, the properties of the mean square projection imply that

$$
\begin{aligned}
\sigma_{X}^{2} & \equiv \mathrm{E}\left(\xi(y, s)-\widehat{\xi_{X}(y, s)}\right)^{2}=\sigma^{2}+\widehat{\sigma_{X}^{2}} \\
& \equiv \mathrm{E}(\xi(y, s)-\widehat{\xi(y, s)})^{2}+\mathrm{E}\left(\widehat{\xi(y, s)}-\widehat{\xi_{X}(y, s)}\right)^{2}
\end{aligned}
$$

for an arbitrary $\widehat{\xi_{X}(y, s)} \in H_{X}$.

Decomposition (2) implies that

$$
\widehat{\xi_{X}(y, s)}=\sum_{k=1}^{N} \alpha_{k} c_{n} \sum_{m \geq 0} \sum_{l=1}^{h(m, n)} S_{m}^{l}\left(\overline{\varphi_{k}}\right) \int_{-\infty}^{\infty} e^{i t_{k} \mu} \zeta_{m}^{l}(r, \mu) d \mu
$$

where $\left(r, \overline{\varphi_{k}}\right)=\left(r, \varphi_{k}^{(1)}, \ldots, \varphi_{k}^{(n-1)}\right)$ are the coordinates of a point $x_{k}$.

Since the random variables $\zeta_{m}^{l}$ are noncorrelated, the interpolation formula (7) for $\widehat{\xi(y, s)}$ together with the addition theorem for spherical harmonics implies that $(15)$

$$
\begin{aligned}
& \widehat{\sigma_{X}^{2}}=\mathrm{E}(\widehat{\xi(y, s)}-\widehat{\xi X(y, s)})^{2} \\
& =c_{n}^{2} \sum_{m \geq 0} \sum_{l=1}^{h(m, n)} \mathrm{E} \mid \sum_{k=1}^{N} \alpha_{k} S_{m}^{l}\left(\overline{\varphi_{k}}\right) \int_{-\infty}^{\infty} e^{i t_{k} \mu} \zeta_{m}^{l}(r, \mu) d \mu \\
& -\left.S_{m}^{l}(\bar{\psi}) \int_{-\infty}^{\infty} \frac{b_{m}(r, \rho, \mu)}{b_{m}(r, \mu)} e^{i s \mu} \zeta_{m}^{l}(r, \mu) d \mu\right|^{2} \\
& =c_{n}^{2} \sum_{m \geq 0} \int_{-\infty}^{\infty} b_{m}(r, \mu) \\
& \times \sum_{l=1}^{h(m, n)}\left\{\sum_{k, j=1}^{N} \alpha_{k} \alpha_{j} S_{m}^{l}\left(\overline{\varphi_{k}}\right) \overline{S_{m}^{l}\left(\overline{\varphi_{j}}\right)}\right. \\
& -\frac{b_{m}(r, \rho, \mu)}{b_{m}(r, \mu)}\left(S_{m}^{l}(\bar{\psi}) e^{i s \mu} \overline{\sum_{k=1}^{N} \alpha_{k} S_{m}^{l}\left(\overline{\varphi_{k}}\right) e^{i t_{k} \mu}}\right. \\
& \left.+\overline{S_{m}^{l}(\bar{\psi}) e^{i s \mu}} \sum_{k=1}^{N} \alpha_{k} S_{m}^{l}\left(\overline{\varphi_{k}}\right) e^{i t_{k} \mu}\right) \\
& \left.+\frac{b_{m}^{2}(r, \rho, \mu)}{b_{m}^{2}(r, \mu)}\left|S_{m}^{l}(\bar{\psi}) e^{i s \mu}\right|^{2}\right\} d \mu \\
& =\frac{c_{n}^{2}}{\omega_{n}} \sum_{m \geq 0} \int_{-\infty}^{\infty} b_{m}(r, \mu)\left\{\sum_{k, j=1}^{N} \alpha_{k} \alpha_{j} e^{i\left(t_{k}-t_{j}\right) \mu} \frac{2 m+n-2}{n-2} C_{m}^{(n-2) / 2}\left(\cos \theta_{k j}\right)\right. \\
& -2 \frac{2 m+n-2}{n-2} \frac{b_{m}(r, \rho, \mu)}{b_{m}(r, \mu)} \sum_{k=1}^{N} \alpha_{k} \cos \left(s-t_{k}\right) C_{m^{\frac{n-2}{2}}}\left(\cos \theta_{k}\right) \\
& \left.+h(m, n) \frac{b_{m}^{2}(r, \rho, \mu)}{b_{m}^{2}(r, \mu)}\right\} d \mu
\end{aligned}
$$

for all $n>2$, where $\theta_{k j}$ and $\theta_{j}$ are the angles between the vectors $x_{k}$ and $x_{j}$ and $x_{j}$ and $y$, respectively, and where $C_{m}^{\nu}(z), m \geq 0$, are the Gegenbauer polynomials defined above. 
We apply the addition theorem for cylindrical functions to the latter result:

$$
\begin{aligned}
\widehat{\sigma_{X}^{2}}= & 2^{(n-2) / 2} \Gamma\left(\frac{n}{2}\right) \sum_{k, j=1}^{N} \alpha_{k} \alpha_{j} \int_{0}^{\infty} \int_{-\infty}^{\infty} \frac{J_{\frac{n-2}{2}}\left(\lambda R_{k j}\right)}{\left(\lambda R_{k j}\right)^{(n-2) / 2}} e^{i\left(t_{k}-t_{j}\right) \mu} f(\lambda, \mu) d \lambda d \mu \\
& -2^{n / 2} \Gamma\left(\frac{n}{2}\right) \sum_{j=1}^{N} \alpha_{j} \int_{0}^{\infty} \int_{-\infty}^{\infty} \frac{J_{\frac{n-2}{2}}\left(\lambda R_{j}\right)}{\left(\lambda R_{j}\right)^{(n-2) / 2}} \cos \left\{\left(s-t_{k}\right) \mu\right\} f(\lambda, \mu) d \lambda d \mu \\
& +2^{n-2} \Gamma^{2}\left(\frac{n}{2}\right) \sum_{m \geq 0} h(m, n) \int_{-\infty}^{\infty} \frac{b_{m}^{2}(r, \rho, \mu)}{b_{m}(r, \mu)} d \mu,
\end{aligned}
$$

where $R_{k j}=2 r \sin \left(\theta_{k j} / 2\right), R_{j}=2 \sqrt{r^{2}+\rho^{2}-2 r \rho \cos \theta_{j}}$, and where $\theta_{k j}$ and $\theta_{j}$ are the angles defined above.

Taking into account the spectral decomposition (11) of the covariance function for a space-isotropic and time-stationary random field, we rewrite the latter result as follows:

$$
\begin{aligned}
\widehat{\sigma_{X}^{2}}= & \sum_{k, j=1}^{N} \alpha_{k} \alpha_{j} \varphi\left(R_{k j}, t_{k}-t_{j}\right)-\sum_{j=1}^{N} \alpha_{j}\left(\varphi\left(R_{j}, s-t_{j}\right)+\overline{\varphi\left(R_{j}, s-t_{j}\right)}\right) \\
& +2^{n-2} \Gamma^{2}\left(\frac{n}{2}\right) \sum_{m \geq 0} h(m, n) \int_{-\infty}^{\infty} \frac{b_{m}^{2}(r, \rho, \mu)}{b_{m}(r, \mu)} d \mu .
\end{aligned}
$$

One of the properties of the covariance function of a homogeneous and isotropic random field is that the matrix $\left(\varphi\left(R_{k j}, t_{k}-t_{j}\right) ; k, j=1, \ldots, N\right)$ generates a quadratic form that is symmetric and positive definite. This allows us to use the criterion for finding the minimum of a positive definite quadratic form and thus to obtain the following result.

In what follows we assume that the function

$$
b_{m}(r, \rho, \mu)
$$

is square integrable on $(-\infty,+\infty)$ with respect to $\mu$.

Theorem 3.1. Let $X=\left\{\left(x_{1}, t_{1}\right), \ldots,\left(x_{N}, t_{N}\right)\right\}$ be a fixed set of observations on an infinite cylindrical surface $E$, that is, $x_{k}=\left(r, \overline{\varphi_{k}}\right)=\left(r, \varphi_{k}^{(1)}, \ldots, \varphi_{k}^{(n-1)}\right)$. The best interpolation in the mean square sense of the random variable $\xi(y, s)$ constructed from observations of $H_{X}(E)$ is given by

$$
\widehat{\xi_{X}(y, s)}=\sum_{k=1}^{N} \alpha_{k} \xi\left(x_{k}, t_{k}\right)
$$

where the vector $\alpha=\left(\alpha_{1}, \ldots, \alpha_{N}\right)$ is a solution of the system

$$
\sum_{k=1}^{N} \alpha_{k} \varphi\left(R_{k j}, t_{k}-t_{j}\right)=\varphi\left(R_{j}, s-t_{j}\right), \quad j=1, \ldots, N
$$

$R_{k j}=2 r \sin \left(\theta_{k j} / 2\right), R_{j}=2 \sqrt{r^{2}+\rho^{2}-2 r \rho \cos \theta_{j}}$, and where $\theta_{k j}$ and $\theta_{j}$ are the angles between the vectors $x_{k}$ and $x_{j}$ and $x_{j}$ and $y$, respectively. 
The corresponding error of interpolation is equal to

$$
\begin{aligned}
\sigma_{X}^{2} \equiv & \mathrm{E}\left(\xi(y, s)-\widehat{\xi_{X}(y, s)}\right)^{2} \\
= & \sigma^{2}+\widehat{\sigma_{X}^{2}} \\
= & \sigma^{2}+2^{n-2} \Gamma^{2}\left(\frac{n}{2}\right) \sum_{m \geq 0} h(m, n) \int_{-\infty}^{\infty} \frac{b_{m}^{2}(r, \rho, \mu)}{b_{m}(r, \mu)} d \mu \\
& -\frac{1}{2} \sum_{j=1}^{N} \alpha_{j} \operatorname{Re}\left\{\varphi\left(R_{j}, s-t_{j}\right)\right\} .
\end{aligned}
$$

Proof. Put

$$
\begin{gathered}
T_{k j}=\varphi\left(R_{k j}, t_{k}-t_{j}\right), \quad t_{j}=\varphi\left(R_{j}, s-t_{j}\right), \\
b=2^{n-2} \Gamma^{2}\left(\frac{n}{2}\right) \sum_{m \geq 0} h(m, n) \int_{-\infty}^{\infty} \frac{b_{m}^{2}(r, \rho, \mu)}{b_{m}(r, \mu)} d \mu .
\end{gathered}
$$

Then

$$
\widehat{\sigma_{X}^{2}}=\alpha^{\prime} T \alpha-\alpha(t+\bar{t})+b .
$$

Thus the necessary and sufficient conditions for the minimum can be written as the following system:

$$
\frac{\partial}{\partial \alpha} \widehat{\sigma_{X}^{2}} \equiv 2 T \alpha-(t+\bar{t})=0
$$

In other words,

$$
0=2 \sum_{k=1}^{N} \alpha_{k} \varphi\left(R_{k j}, t_{k}-t_{j}\right)-\left(\varphi\left(R_{j}, s-t_{j}\right)+\overline{\varphi\left(R_{j}, s-t_{j}\right)}\right) .
$$

Then the corresponding error is equal to

$$
\widehat{\sigma_{X}^{2}}=\alpha^{\prime} T \alpha-\alpha(t+\bar{t})+b=\frac{1}{2} \alpha(t+\bar{t})-\alpha(t+\bar{t})+b=b-\frac{1}{2} \alpha(t+\bar{t}),
$$

and this completes the proof.

\section{LIMIT THEOREM FOR THE ERROR OF INTERPOLATION}

One can use a certain approximation if the exact solution of the system of equations (18) is hard to evaluate.

Let, for $t \in(-\infty,+\infty)$,

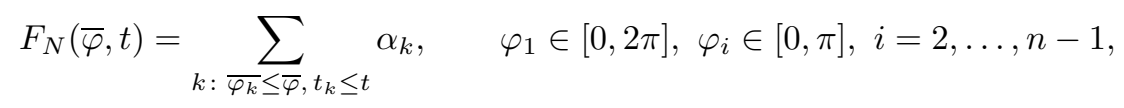

denote the cumulative distribution function of the set of coefficients $\left\{\alpha_{1}, \ldots, \alpha_{N}\right\}$. The inequality $\overline{\varphi_{k}} \leq \bar{\varphi}$ in the above notation means that the system of inequalities $\varphi_{k}^{(i)} \leq \varphi_{i}$, $i=1, \ldots, n-1$, holds. 
Considering (15), we deduce that

$$
\begin{aligned}
& \widehat{\sigma_{X}^{2}}=c_{n}^{2} \sum_{m \geq 0} \int_{-\infty}^{\infty} b_{m}(r, \mu) \sum_{l=1}^{h(m, n)}\left\{\int_{-\infty}^{\infty} \int_{-\infty}^{\infty} \int_{\Pi_{n}} \int_{\Pi_{n}} S_{m}^{l}(\bar{\varphi}) \overline{S_{m}^{l}(\bar{\psi})} e^{i t \mu} e^{-i s \mu} d F_{N}(\bar{\varphi}, t) d F_{N}(\bar{\psi}, s)\right. \\
& \quad-\frac{b_{m}(r, \rho, \mu)}{b_{m}(r, \mu)}\left(S_{m}^{l}(\bar{\psi}) e^{i s \mu} \int_{-\infty}^{\infty} \int_{\Pi_{n}} \overline{S_{m}^{l}(\bar{\varphi})} e^{-i t \mu} d F_{N}(\bar{\varphi}, t)\right. \\
&\left.+\overline{S_{m}^{l}(\bar{\psi})} e^{-i s \mu} \int_{-\infty}^{\infty} \int_{\Pi_{n}} S_{m}^{l}(\bar{\varphi}) e^{i t \mu} d F_{N}(\bar{\varphi}, t)\right) \\
&=c_{n}^{2} \sum_{m \geq 0} \int_{-\infty}^{\infty} b_{m}(r, \mu)\left.\frac{b_{m}^{2}(r, \rho, \mu)}{b_{m}^{2}(r, \mu)}\left|S_{m}^{l}(\bar{\psi})\right|^{2}\right\} d \mu \\
& \times \sum_{l=1}^{h(m, n)}\left|\int_{-\infty}^{\infty} \int_{\Pi_{n}} S_{m}^{l}(\bar{\varphi}) e^{i t \mu} d F_{N}(\bar{\varphi}, t)-\frac{b_{m}(r, \rho, \mu)}{b_{m}(r, \mu)} S_{m}^{l}(\bar{\psi}) e^{i s \mu}\right|^{2},
\end{aligned}
$$

where $\Pi_{n}=[0,2 \pi] \times \underbrace{[0, \pi] \times \cdots \times[0, \pi]}_{n-2}$.

Theorem 4.1. Assume that a sequence of functions $F_{N}(\cdot, \cdot)$ have a uniformly bounded variation on $\Pi_{n} \times(-\infty,+\infty)$. We further assume that this sequence weakly converges to the function $F(\cdot, \cdot)$ with bounded variation. Then the limit mean square error of the approximation of the integral $\widehat{\xi(y, s)}$ is equal to

$$
\widehat{\sigma_{\infty}^{2}}=c_{n}^{2} \sum_{m \geq 0} \int_{-\infty}^{\infty} b_{m}(r, \mu) \sum_{l=1}^{h(m, n)}\left|\int_{-\infty}^{\infty} \int_{\Pi_{n}} S_{m}^{l}(\bar{\varphi}) e^{i t \mu} d F(\bar{\varphi}, t)-e^{i s \mu} c_{m}^{l}(y, \mu)\right|^{2} d \mu
$$

where

$$
c_{m}^{l}(y, \mu)=\frac{b_{m}(r, \rho, \mu)}{b_{m}(r, \mu)} S_{m}^{l}(\bar{\psi}) .
$$

Proof. The result follows by passing to the limit in (20) as $N \rightarrow \infty$ in view of the continuity of the Bessel function and by the definition of the convergence of functions with bounded variations.

Corollary 4.1. If the assumptions of Theorem 4.1 hold, then the asymptotic error of interpolation is equal to zero if and only if

$$
\int_{-\infty}^{\infty} \int_{\Pi_{n}} S_{m}^{l}(\bar{\varphi}) e^{i t \mu} d F(\bar{\varphi}, t)=e^{i s \mu} c_{m}^{l}(y, \mu)
$$

for all $m \geq 0, l=1, \ldots, h(m, n)$, and $\mu \in(-\infty,+\infty)$.

Corollary 4.2. If

$$
\sum_{m \geq 0} \sum_{l=1}^{h(m, n)}\left|c_{m}^{l}(y, \mu)\right|^{2}<+\infty, \quad \mu \in(-\infty,+\infty)
$$


then the asymptotic error of interpolation is equal to zero if and only if the limit density is such that

$$
F^{\prime}(\bar{\varphi}, t)=\frac{l(\bar{\varphi})}{2 \pi} \int_{-\infty}^{\infty} e^{i(s-t) \nu} C(y, x, \nu) d \nu
$$

for $\bar{\varphi} \in \Pi_{n}, t \in(-\infty,+\infty)$, where

$$
\begin{gathered}
l(\bar{\varphi})=\left(\sin \varphi_{2}\right)^{n-2}\left(\sin \varphi_{3}\right)^{n-3} \cdots \sin \varphi_{n-1}, \\
C(y, x, \nu)=\frac{1}{\omega_{n}(n-2)} \sum_{m \geq 0} \frac{b_{m}(r, \rho, \nu)}{b_{m}(r, \nu)}(2 m+n-2) C_{m}^{(n-2) / 2}(\cos \theta),
\end{gathered}
$$

and where $\theta$ denotes the angle between the vectors $\{r, \bar{\varphi}, 0\}$ and $\{\rho, \bar{\psi}, 0\}$, while $C_{m}^{\nu}(z)$, $m \geq 0$, are the Gegenbauer polynomials defined by (11).

Proof. Note that the integrals

$$
\begin{aligned}
\int_{\Pi_{n}} S_{m}^{l}(\bar{\varphi}) \int_{-\infty}^{\infty} e^{i t \mu} d F(\bar{\varphi}, t) & =\int_{\Pi_{n}} S_{m}^{l}(\bar{\varphi}) \int_{-\infty}^{\infty} e^{i t \mu} l(\bar{\varphi}) \frac{d F(\bar{\varphi}, t)}{l(\bar{\varphi})} \\
& =\int_{S_{n}} S_{m}^{l}(\bar{\varphi}) \int_{-\infty}^{\infty} e^{i t \mu} \frac{d F(\bar{\varphi}, t)}{l(\bar{\varphi})} d \mu_{n}(\bar{\varphi})
\end{aligned}
$$

are the generalized Fourier coefficients of the function

$$
\int_{-\infty}^{\infty} e^{i t \mu} \frac{d F(\bar{\varphi}, t)}{l(\bar{\varphi})}
$$

with respect to the system of functions $\left\{S_{m}^{l}(\bar{\varphi})\right\}_{m \geq 0, l=1, \ldots, h(m, n)}$ that are orthonormal on the sphere $S_{n}$. Assume that the limit distribution function $F(\bar{\varphi}, t)$ is absolutely integrable, that is, the density $F^{\prime}(\bar{\varphi}, t)$ exists. Then Corollary 4.1 and condition (23) imply that

$$
\begin{aligned}
\int_{-\infty}^{\infty} e^{i t \mu} F^{\prime}(\bar{\varphi}) d t & =l(\bar{\varphi}) \cdot \sum_{m \geq 0} \sum_{l=1}^{h(m, n)} \frac{b_{m}(r, \rho, \mu)}{b_{m}(r, \mu)} S_{m}^{l}(\bar{\psi}) S_{m}^{l}(\bar{\varphi}) e^{i s \mu} \\
& =l(\bar{\varphi}) e^{i s \mu} \cdot \sum_{m \geq 0} \frac{b_{m}(r, \rho, \mu)}{b_{m}(r, \mu)} \frac{h(m, n)}{\omega_{n}} \frac{C_{m}^{(n-2) / 2}(\cos \theta)}{C_{m}^{(n-2) / 2}(1)} \\
& =\frac{l(\bar{\varphi})}{\omega_{n}} \sum_{m \geq 0} \frac{b_{m}(r, \rho, \mu)}{b_{m}(r, \mu)} \\
& \times \frac{(2 m+n-2)(m+n-3) ! m !(n-3) !}{(n-2) ! m !(m+n-3) !} C_{m}^{(n-2) / 2}(\cos \theta) \\
= & l(\bar{\varphi}) \cdot C(y, x, \mu)
\end{aligned}
$$

by the addition theorem for spherical harmonics.

Applying the inversion formula for the Fourier transform we complete the proof of Corollary 4.2 ,

Corollary 4.3. Let

$$
X_{N}=\left\{\left(x_{1}, t_{1}\right), \ldots,\left(x_{N}, t_{N}\right)\right\}
$$

be an arbitrary sequence of sets, where $x_{k}=\left(r, \overline{\varphi_{k}}\right)$,

$$
0<\varphi_{1}^{(1)}<\varphi_{2}^{(1)}<\cdots<\varphi_{N}^{(1)}=2 \pi, \quad 0<\varphi_{1}^{(i)}<\varphi_{2}^{(i)}<\cdots<\varphi_{N}^{(i)}=\pi
$$

for $i=2, \ldots, n-1$ and $t_{k} \in(-\infty,+\infty)$. 
Assume that condition (23) holds. If the coefficients

$$
\alpha_{k}=l\left(\bar{\varphi}_{k}\right) \int_{-\infty}^{\infty} e^{-i t_{k} \nu} C\left(y, x_{k}, \nu\right) d \nu \Delta \overline{\varphi_{k}} \Delta t_{k}
$$

where $\Delta \overline{\varphi_{k}}:=\prod_{i=1}^{n-1}\left(\varphi_{k}^{(i)}-\varphi_{k-1}^{(i)}\right)$, are such that

$$
\max _{k=1, \ldots, N}\left|\prod_{i=1}^{n-1}\left(\varphi_{k}^{(i)}-\varphi_{k-1}^{(i)}\right)\right| \rightarrow 0
$$

and $\max _{k=1, \ldots, N}\left|\Delta t_{k}\right| \rightarrow 0$ as $N \rightarrow \infty$, then the mean square error of approximation $\widehat{\sigma_{X}^{2}}$ approaches zero as $N \rightarrow \infty$.

Proof. Taking into account representation (15), the mean square error of interpolation is equal to

$$
\widehat{\sigma_{X}^{2}}=c_{n}^{2} \sum_{m \geq 0} \int_{-\infty}^{\infty} b_{m}(r, \mu) \sum_{l=1}^{h(m, n)}\left|\int_{-\infty}^{\infty} \int_{\Pi_{n}} e^{i t \mu} S_{m}^{l}(\bar{\varphi}) d F_{N}(\bar{\varphi}, t)-e^{i s \mu} c_{m}^{l}(y, \mu)\right|^{2} d \mu .
$$

On the other hand,

$$
\widehat{\sigma_{X}^{2}}=c_{n}^{2} \sum_{m \geq 0} \int_{-\infty}^{\infty} b_{m}(r, \mu) \sum_{l=1}^{h(m, n)}\left|\sum_{k=1}^{N} \alpha_{k} S_{m}^{l}\left(\overline{\varphi_{k}}\right) e^{i t_{k} \mu}-e^{i s \mu} c_{m}^{l}(y, \mu)\right|^{2} d \mu .
$$

Now we substitute the coefficients $\alpha_{k}$ to the latter expression for $\widehat{\sigma_{X}^{2}}$ and obtain

$$
\begin{aligned}
c_{n}^{2} \sum_{m \geq 0} b_{m}(r, \mu) \sum_{l=1}^{h(m, n)}\left|\int_{-\infty}^{\infty} \int_{\Pi_{n}} e^{i t \mu} S_{m}^{l}(\bar{\varphi}) d F_{N}(\bar{\varphi}, t)-e^{i s \mu} c_{m}^{l}(y, \mu)\right|^{2} d \mu \\
=\frac{c_{n}^{2}}{2 \pi} \sum_{m \geq 0} b_{m}(r, \mu) \sum_{l=1}^{h(m, n)} \mid \sum_{k=1}^{N} \int_{-\infty}^{\infty} S_{m}^{l}\left(\overline{\varphi_{k}}\right) l\left(\bar{\varphi}_{k}\right) \int_{-\infty}^{\infty} e^{-i t_{k} \nu} C\left(y, x_{k}, \nu\right) \\
\times e^{i s \nu} d \nu \Delta \overline{\varphi_{k}} \Delta t_{k}-\left.e^{i s \mu} c_{m}^{l}(y, \mu)\right|^{2}
\end{aligned}
$$

Passing to the limit on both sides of the latter equality as $N \rightarrow \infty$, we get

$$
\begin{aligned}
\int_{-\infty}^{\infty} & \int_{S_{n}} e^{i t \mu} S_{m}^{l}(\bar{\varphi}) \frac{F^{\prime}(\bar{\varphi}, t)}{l(\bar{\varphi})} d \mu_{n} d t \\
& =\int_{-\infty}^{\infty} \int_{S_{n}} e^{i t \mu} S_{m}^{l}(\bar{\varphi}) \frac{1}{2 \pi} \int_{-\infty}^{\infty} e^{-i t \nu} C(y, x, \nu) e^{i s \nu} d \nu d \mu_{n}(\bar{\varphi}) d t
\end{aligned}
$$

for all $l=1, \ldots, h(m, n), m \geq 0$, and $\mu \in(-\infty,+\infty)$.

Since the system of functions $\left\{S_{m}^{l}(\cdot), l=1, \ldots, h(m, n), m \geq 0\right\}$ is complete on the sphere $S_{n}$, we deduce from the uniqueness of the Fourier transform that

$$
\frac{F^{\prime}(\bar{\varphi}, t)}{l(\bar{\varphi})} \equiv \frac{1}{2 \pi} \int_{-\infty}^{\infty} e^{-i t \nu} C(y, x, \nu) e^{i s \nu} d \nu
$$

Thus Corollary 4.2 implies the result desired. 


\section{CONCLUding REMARKS}

We considered the interpolation of a homogeneous and isotropic random field for an arbitrary point in the case of a finite set of observations on an infinite cylindrical surface. We found a solution of this problem as well as an explicit expression for the corresponding mean square error. The asymptotic behavior of the error is studied as the number of observations increases. We also discussed the conditions for the error-free approximation. We found the optimal distribution of weight coefficients of the interpolation.

The results for random fields with density belonging to a certain class of functions will be published elsewhere.

\section{BIBLIOGRAPHY}

1. M. I. Yadrenko, Spectral Theory of Random Fields, Vyshcha shkola, Kiev, 1980; English transl., Optimization Software, Inc., Publications Division, New York, 1983. MR590889 (82e:60001)

2. Yu. D. Popov, Some problems of linear extrapolation for homogeneous, space isotropic, and time stationary random fields, Dopovidi Akad. Nauk Ukrain. RSR Ser. A (1968), no. 12, 166-177. (Russian)

3. M. V. Kartashov, Finite-dimensional interpolation of a random field on the plane, Teor. Imovir. Mat. Stat. 51 (1994), 53-61; English transl. in Theory Probab. Math. Statist. 51 (1995), 53-61. MR 1445052 (97k:60142)

4. N. Semenovs'ka, A problem of the interpolation of a homogeneous and isotropic random field, Teor. Imovir. Mat. Stat. 74 (2006), 150-158; English transl. in Theory Probab. Math. Statist. $\mathbf{7 4}$ (2007), 171-179. MR2336787 (2008g:60157)

Department of Probability Theory, Statistics, and Actuarial Mathematics, Faculty for Mechanics and Mathematics, National Taras Shevchenko University, Academician Glushkov Avenue 2, Kiev 03127, Ukraine

E-mail address: semenovska@mail.ru

Received 18/JAN/2010

Translated by OLEG KLESOV 